\title{
Low-frequency and trend compensation of broadband seismograms
}

\author{
Shigeo Kinoshita \\ Yokohama City University, Seto 22-2, Kanazawa-ku, Yokohama 236-0027, Japan \\ (Received July 11, 2011; Revised February 5, 2012; Accepted February 15, 2012; Online published April 16, 2012)
}

\begin{abstract}
The present paper proposes a technique for low-frequency and trend compensation of broadband seismograms, which involves frequency-band broadening using digital filtering and background-trend compensation based on a Heaviside-type tilt signal estimated using a stochastic trend model. Applying this method to the east-west component of broadband seismograms, recorded using VSE355G3 broadband seismometers at the KSN site of F-net for the 2011 off the Pacific coast of Tohoku Earthquake $\left(M_{\mathrm{w}} 9.0\right)$, allowed a sharpened velocity waveform to be obtained and the static displacement associated with the earthquake to be determined.
\end{abstract}

Key words: Broadband seismograms, strong motion, band broadening, digital compensation filter, background trend.

\section{Introduction}

Since broadband seismograms are useful in earthquake source studies, many broadband networks, such as Fnet (http://www.fnet.bosai.go.jp/) in Japan, are operated throughout the world. The recent development of the Internet enables published data to be analyzed soon after the occurrence of large earthquakes such as the 2011 off the Pacific coast of Tohoku Earthquake (hereafter, referred to as the 2011 Tohoku Earthquake). The standard frequency band of broadband seismometers is from 0.01 (or 0.003 ) to approximately $100 \mathrm{~Hz}$. Thus, we have to compensate for the low-frequency components in order to fully understand the seismic behavior associated with such large earthquakes. In addition, the background trend caused by tilt motion must be removed in order to analyze the low-frequency components of broadband seismograms.

GEONET, the Global Positioning System (GPS) established by the Geographical Survey Institute (GSI), recorded land deformation up to approximately 5 meters on the Pacific coast of eastern Honshu (http://terras.gsi.go.jp/ja/ index.html) following the 2011 Tohoku Earthquake: an east-southeast deformation of 5.3 meters and a downward deformation of 1.2 meters were measured at the Ojika GPS site. Such a permanent coseismic displacement, i.e., a nearfield static displacement, and its spatial distribution can lead to a tilt step in near-field acceleration signals as shown by Pillet and Virieux (2007). An estimation of static coseismic displacements using strong-motion accelerograms, was conducted by Boore (2001) and Wu and Wu (2007) for the 1999 Chi-Chi, Taiwan Earthquake, and Javelaud et al. (2011) for the 2007 Niigata-ken Chuetsu-Oki Earthquake. Kinoshita and Takagishi (2011) also demonstrated the generation and propagation of static displacement during the

Copy right (C) The Society of Geomagnetism and Earth, Planetary and Space Sciences (SGEPSS); The Seismological Society of Japan; The Volcanological Society of Japan; The Geodetic Society of Japan; The Japanese Society for Planetary Sciences; TERRAPUB.

doi:10.5047/eps.2012.02.002
2011 Tohoku Earthquake, using KiK-net borehole recordings (http://www.kik.bosai.go.jp/), and showed that the resulting static displacements were in good agreement with land deformations determined from GPS data. The KiKnet borehole recordings were measured using negative feedback accelerometers with a frequency band of 0 to approximately $30 \mathrm{~Hz}$, so estimation of the static displacement is possible without any frequency compensation.

Although broadband seismometers have no sensitivity at zero frequency, estimation of the static displacement may be possible by compensating for the low-frequency components. Of course, the estimated static displacement is coseismic behavior in the analyzed data window. To verify that such an estimation is possible using broadband seismograms, this paper proposes a technique for implementing low-frequency and trend compensation of broadband seismograms.

\section{VSE355G3 Broadband Seismogram and Frequency-Compensation Filter}

Broadband seismometer networks are equipped with negative feedback seismometers, as represented by the block diagram shown in Fig. 1. The two built-in feedback transfer functions, $G_{\mathrm{M}}(s)$ and $G_{\mathrm{A}}(s)$, are the main differentiation and auxiliary integration circuit elements, respectively, and $s$ is a Laplace variable. In high-sensitivity broadband seismometers, such as an STS-2, the main feedback circuit uses passive elements; whereas in low-sensitivity seismometers such as a VSE355G3, active elements are used. F-net stations are equipped with both kinds of seismometers. The overall transfer function of the seismometer represented in Fig. 1 is given by

$$
G(s)=\frac{s G_{\mathrm{O}}(s)}{1+G_{\mathrm{O}}(s)\left[G_{\mathrm{M}}(s)+G_{\mathrm{A}}(s)\right]}[\mathrm{V} /(\mathrm{m} / \mathrm{s})]
$$

against the input velocity signal, where $G_{\mathrm{O}}(s)$ is the transfer function of the mass-spring system with a position detector for mass movement. This article deals only with seismograms measured by VSE355G3 broadband seismometers 


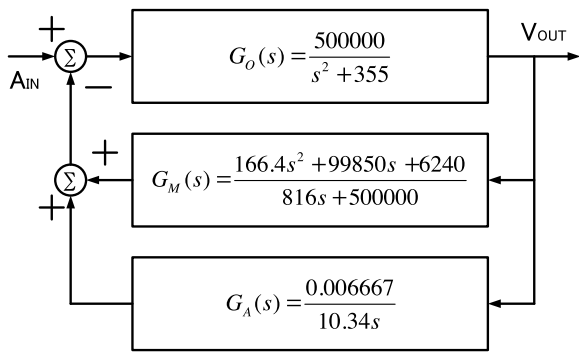

Fig. 1. Block diagram of VSE355G3 broadband seismometer; output velocity $V_{\text {OUT }}(s)$ against input acceleration $A_{\mathrm{IN}}(s)$ in units of $\mathrm{V} / \mathrm{m} / \mathrm{s}^{2}$.
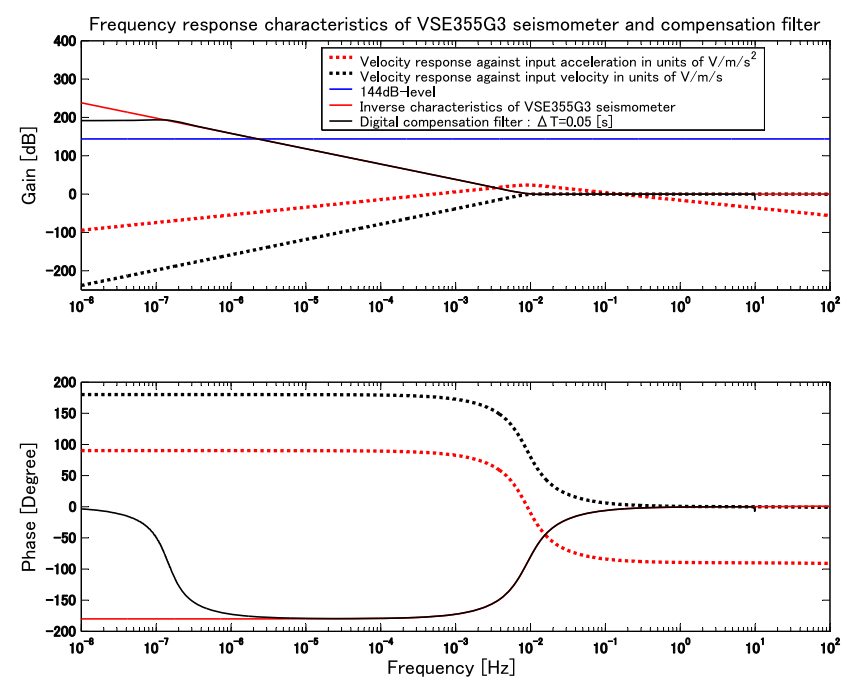

Fig. 2. Frequency response characteristics of VSE355G3 broadband seismometer and compensation filter used in this study; relative velocity response of VSE355G3 seismometer against input acceleration (dotted red line), that against input velocity (dotted black line), inverse frequency response of VSE355G3 seismometer (red line), $144 \mathrm{~dB}$ level (blue line) and frequency response of digital compensation filter used in this study (black line) with a sampling time of $1 / \Delta T=0.05 \mathrm{~s}$.

(Hutt et al., 2008), since the seismic signals from the highsensitivity seismometers exceeded the clipping level of the instruments during the 2011 Tohoku Earthquake.

The numerical values in Fig. 1 correspond to the VSE355G3 seismometers used by F-net. The frequency characteristics of these seismometers are shown by the dotted black line in Fig. 2. Broadband recordings measured by the seismometers are published on the Internet by F-net at a sampling frequency of $20 \mathrm{~Hz}$ (http://www.fnet.bosai.go.jp). The high-frequency components of the recordings are characterized by a low-pass decimation filter with a pass-band corner frequency of approximately $9 \mathrm{~Hz}$, and a stop-band corner frequency of exactly $9.7 \mathrm{~Hz}$. These corner frequencies are easily recognized using the published data in the Fourier domain. Since the Nyquist frequency is $10 \mathrm{~Hz}$, no high-frequency compensation is required when analyzing VSE355G3 seismograms. The low-frequency components of the recordings are characterized by a corner frequency of approximately $0.01 \mathrm{~Hz}$, as shown by the dotted black line in Fig. 2. Thus, in the case of a 24-bit acquisition system, it may be possible to compensate for the lowfrequency components until the inverse frequency response of the VSE355G3 becomes $144 \mathrm{~dB}$ relative to the gain level in the flat frequency response of the seismometer. This lowest possible compensation frequency is indicated by the intersection of the red and blue lines in Fig. 2: the red line represents the inverse frequency characteristics and the blue line is the $144 \mathrm{~dB}$ level.

Thus, the frequency band whose in-band frequencies are lower than the lowest compensation frequency is the socalled "Don't care region", and we can arbitrarily alter the inverse response of the VSE355G3 seismometer to implement stable compensation of the seismograms. We therefore propose the use of a digital compensation filter whose frequency characteristics are shown by the black line in Fig. 2. This is the default compensation filter. In this study, the digital filter given by Eq. (2), that is designed by slightly modifying the inverse characteristics of a VSER355G3 seismometer, is used with a sampling frequency of $20 \mathrm{~Hz}$ for low-frequency compensation. Of course, the zeros and poles of this digital compensation filter are inside the unit circle in the $z$-plane.

$$
\begin{aligned}
& H(z)=\frac{\sum_{k=0}^{4} a_{k} z^{k}}{1+\sum_{k=1}^{4} b_{k} z^{k}}, \\
& \text { where }\left[\begin{array}{l}
a_{0} \\
a_{1} \\
a_{2} \\
a_{3} \\
a_{4}
\end{array}\right]=\left[\begin{array}{r}
1.00177927805847 \\
-0.12272121268339 \\
-1.87452571371945 \\
0.12254024864872 \\
0.87295766147310
\end{array}\right] \text { and } \\
& {\left[\begin{array}{l}
b_{1} \\
b_{2} \\
b_{3} \\
b_{4}
\end{array}\right]=\left[\begin{array}{r}
-0.12755928712526 \\
-1.87182784134148 \\
0.12633369387852 \\
0.87305343458823
\end{array}\right] .}
\end{aligned}
$$

\section{Trend Estimation}

Kinoshita and Takagishi (2011) demonstrated the generation and propagation of static displacement, using KiKnet borehole recordings, for the 2011 Tohoku Earthquake, by compensating for the trend produced in velocity seismograms calculated from the original KiK-net accelerograms. In their study, the trend was assumed to be due to tilt motion during earthquake faulting. Thus, the evaluation of broadband seismograms requires a trend-compensation process for velocity signals output from a digital compensation filter. Moving average or polynomial fitting methods are frequently used for compensating for trend signals, which correspond to long-term movement of random signals. In recent trend-estimation studies, a popular signalprocessing approach has been time-series modeling of trend signals, since such stochastic models are highly flexible and can address trend signals more objectively (Kitagawa and Gersch, 1996; Kitagawa, 2010). Thus, in the present study, a stochastic trend model based on linear fitting is used. This method is implemented using a state space representation of the trend signal (Kitagawa, 2010):

$$
\begin{aligned}
& x_{n}=F x_{n-1}+\varepsilon_{n}, x_{n}=\left[\begin{array}{c}
T_{n} \\
T_{n-1}
\end{array}\right], F=\left[\begin{array}{rr}
2 & -1 \\
1 & 0
\end{array}\right] \text { and } \\
& E\left[\varepsilon_{n} \varepsilon_{m}\right]=\delta_{m n} \sigma_{\varepsilon}^{2} \text { for } n=1,2, \ldots, N,
\end{aligned}
$$


where the system noise $\left\{\varepsilon_{n}\right\}_{n=1}^{N}$ is assumed to be Gaussian white noise with a covariance of $\sigma_{\varepsilon}^{2}$. The frequencycompensated velocity signal $\left\{v_{n}\right\}_{n=1}^{N}$ is represented using the trend signal $\left\{T_{n}\right\}_{n=1}^{N}$ and the observation noise $\left\{w_{n}\right\}_{n=1}^{N}$, which is assumed to be Gaussian white noise with a covariance of $\sigma_{w}^{2}$, as follows:

$$
\begin{aligned}
& v_{n}=T_{n}+w_{n}=H x_{n}+w_{n}, H=\left[\begin{array}{ll}
1 & 0
\end{array}\right] \text { and } \\
& E\left[w_{n} w_{m}\right]=\delta_{m n} \sigma_{w}^{2} \text { for } n=1,2, \ldots, N .
\end{aligned}
$$

In this study, $N \Delta T=400 \mathrm{~s}$ is the length of the data window used for modeling the trend signal in the S-coda part and $\Delta T$ is the sampling time of $0.05 \mathrm{~s}$. Based on Kalman filtering (Kalman, 1960; Lee, 1964):

$$
\begin{aligned}
& x_{n \mid n-1}=F x_{n-1 \mid n-1}, \\
& V_{n \mid n-1}=F V_{n-1 \mid n-1} F^{\prime}+\sigma_{\varepsilon}^{2}, \\
& K_{n}=V_{n \mid n-1} H^{\prime}\left(H V_{n \mid n-1} H^{\prime}+\sigma_{w}^{2}\right)^{-1} \\
& x_{n \mid n}=x_{n \mid n-1}+K_{n}\left(v_{n}-H x_{n \mid n-1}\right) \text { and } \\
& V_{n \mid n}=\left(I-K_{n} H\right) V_{n \mid n-1}, \text { for } n=1,2, \ldots, N,
\end{aligned}
$$

a fixed-interval Kalman smoother (Lee, 1964; Rauch et al., $1965)$ is implemented to produce a smoothed trend signal $\left\{\hat{T}_{n}=\right.$ first element of vector $\left.x_{n \mid N}\right\}$ as follows:

$$
\begin{aligned}
& A_{n}=V_{n \mid n} F^{\prime} V_{n+1 \mid n}^{-1}, \\
& x_{n \mid N}=x_{n \mid n}+A_{n}\left(x_{n+1 \mid N}-x_{n+1 \mid n}\right) \text { and } \\
& V_{n \mid N}=V_{n \mid n}+A_{n}\left(V_{n+1 \mid N}-V_{n+1 \mid n}\right) A_{n}^{\prime}, \\
& \text { for } n=N-1, \ldots, 2,1 .
\end{aligned}
$$

In the above recursive relations, matrices $X^{\prime}$ and $X^{-1}$ are the transposed matrix of $X$ and the inverse matrix of $X$, respectively, and $I$ is the identity matrix. The initial conditions for the recursive calculations are given by

$$
\begin{aligned}
& x_{0 \mid 0}=\left[\begin{array}{c}
\sum_{n=1}^{N} v_{n} \equiv \bar{v} \\
0
\end{array}\right], V_{0 \mid 0}=\left[\begin{array}{cc}
\sigma_{\varepsilon}^{2} & 0 \\
0 & \sigma_{\varepsilon}^{2}
\end{array}\right] \text { and } \\
& \sigma_{w}^{2}=\frac{1}{N} \sum_{n=1}^{N}\left(v_{n}-\bar{v}\right)^{2} .
\end{aligned}
$$

Although the covariance of the system noise, $\sigma_{\varepsilon}^{2}$, is an unknown parameter, a Kalman filter gives the likelihood of the trend model for a given $\sigma_{\varepsilon}^{2}$, so that the value of $\sigma_{\varepsilon}^{2}$ can be determined using an Akaike information criterion (AIC) minimization procedure (Kitagawa, 2010). In the following example, the value of $1 / 2^{30}$ is assigned to $\sigma_{\varepsilon}^{2}$ as a result. Finally, by fitting a straight line to the $\mathrm{S}$-coda part of the estimated $\left\{\hat{T}_{n}\right\}_{n=1}^{N}$, we determine a Heaviside-type tilt signal $a_{\text {tilt }}(t)=\alpha U\left(t-t_{\text {onset }}\right)$ in the acceleration domain, where $U(t)$ is a unit step function. The two parameters $\left\{\alpha, t_{\text {onset }}\right\}$ are determined from the gradient of the fitted straight line and the time point at which the estimated line intersects the baseline of the frequency-compensated velocity signal, respectively.

\section{Example}

We will now show an example of low-frequency and trend compensation of a broadband seismogram: the eastwest component seismogram recorded by the VSE355G3

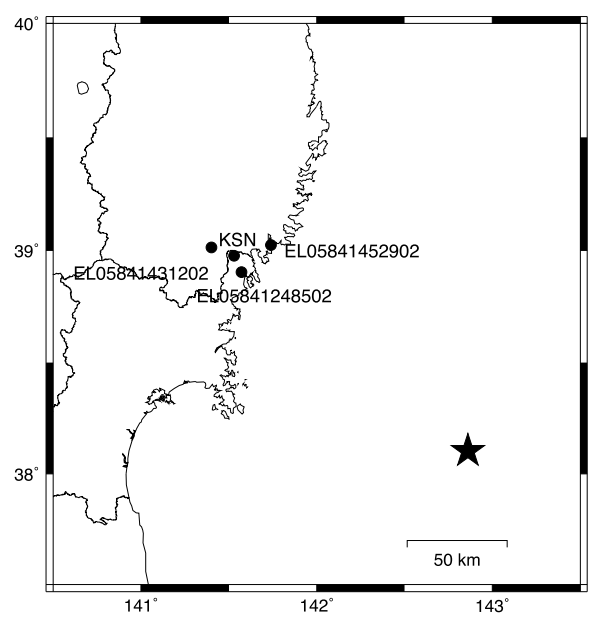

Fig. 3. Locations of the KSN site and three GPS sites of GEONET. The epicenter of the 2011 Tohoku Earthquake is shown by a solid star symbol.

seismometer at the $\mathrm{KSN}$ site $\left(38.9762^{\circ} \mathrm{N}, 141.5301^{\circ} \mathrm{N}\right)$ of F-net during the 2011 Tohoku Earthquake (Fig. 3). The original velocity seismogram is shown in Fig. 4(a). The frequency-compensated seismogram obtained using the digital compensation filter described by Eq. (2) is shown by a black line in Fig. 4(b). The red line is the smoothed trend signal produced by the stochastic trend model for a data window from 500 to $900 \mathrm{~s}$. By differentiating the smoothed trend signal, the velocity gradient signal is calculated. Figure 4(c) is an enlarged portion of the velocity gradient signal, which reveals a stable state with an approximately constant velocity gradient of $-8.6 \times 10^{-5}$ $\mathrm{m} / \mathrm{s}^{2}$. Thus, by fitting a straight line to the smoothed trend signal in the data window from 550 to $850 \mathrm{~s}$, we obtain $a_{\mathrm{tilt}}(t)=\alpha U\left(t-t_{\text {onset }}\right)$, where $\alpha=-8.6 \times 10^{-5} \mathrm{~m} / \mathrm{s}^{2}$ and $t_{\text {onset }}=159.75 \mathrm{~s}$. The smoothed trend signal and its piecewise linear trend model calculated from $a_{\text {tilt }}(t)$ are shown in Fig. 4(d) by red and black lines, respectively. The frequency- and trend-compensated east-west velocity seismogram is shown in Fig. 4(e). We find that a sharpened velocity waveform is obtained by broadening the original frequency band of the broadband seismogram. Finally, we can calculate the displacement waveform by integrating the frequency- and trend-compensated velocity signal as shown in Fig. 4(f). Three GEONET stations that closely surround the KSN site $(38.9762 \mathrm{~N}, 141.5301 \mathrm{E})$ are the EL05841431202 (39.0121N, 141.4009E), EL05841248502 $(38.9028 \mathrm{~N}, 141.5726 \mathrm{E})$ and EL05841452902 (39.0238N, 141.7399E) stations (Fig. 3). The horizontal displacements measured at these GPS sites just after the earthquake were $3.30,4.12$ and $4.19 \mathrm{~m}$, respectively, mostly in an easterly direction, which are in good agreement with the estimated horizontal static displacement of $3.62 \mathrm{~m}$ at the KSN site. Of course, the estimates of the offset acceleration $\alpha$ and the static displacement depend on the corner frequency of the compensation filter. Strictly speaking, the corner frequency must be determined by taking account of the $1 / f$ noise behavior (Javelaud et al., 2011): the noise power increases linearly with the period. Although the low-frequency noise model for the F-net recording system with VSE355G3 seis- 


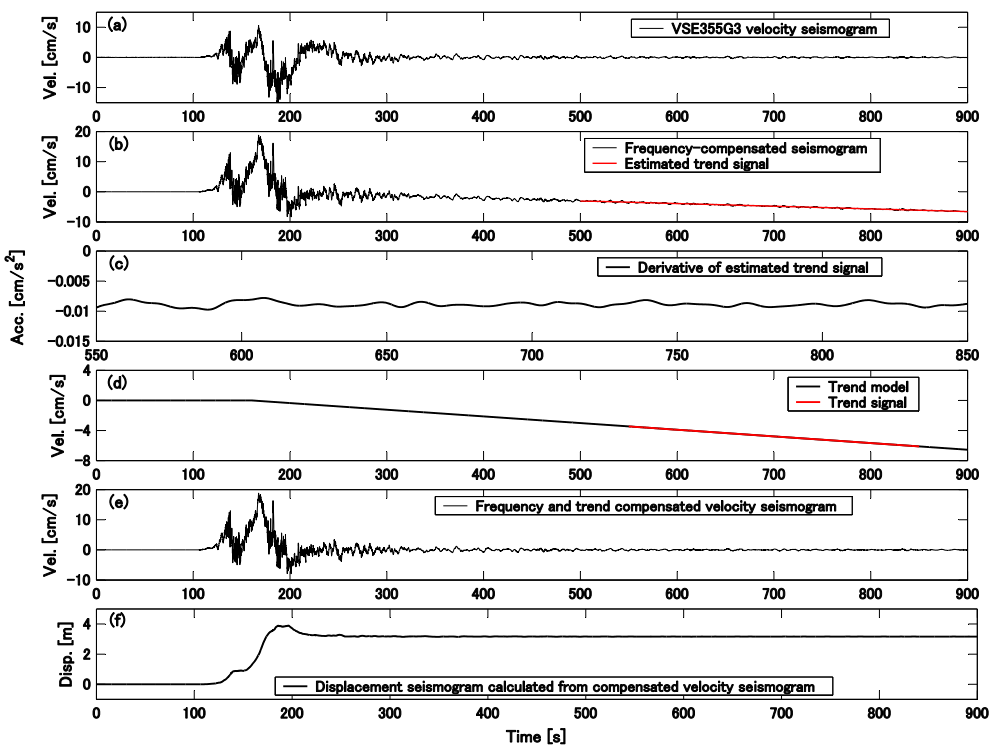

Fig. 4. Example of low-frequency and trend-compensated broadband seismogram and estimated displacement; (a) original east-west component seismogram recorded at the KSN site, (b) frequency-compensated seismogram (black line) and estimated trend signal (red line), (c) derivative of estimated trend signal shown by a red line in (b), (d) piecewise linear trend model (black line) used for trend compensation in the seismogram shown in (b) and linear fit (red line) to the estimated trend signal shown in (b), (e) frequency- and trend-compensated seismogram, and (f) displacement seismogram calculated from the velocity seismogram shown in (e).

mometers is unknown, the present method makes it possible to adjust the range of corner frequencies by comparison with the coseismic displacements measured at three GPS sites close to the KSN site. The results indicate that the static displacements estimated using a corner frequency of less than $5 \times 10^{-6} \mathrm{~Hz}$ are within the range of the three GPSdetermined coseismic displacements; the flat level of the compensation filter at low frequencies is higher than $124 \mathrm{~dB}$ in Fig. 2, and the acceleration offset estimated using a compensation filter with a corner frequency of $5 \times 10^{-6} \mathrm{~Hz}$ is $\alpha=-8.3 \times 10^{-5} \mathrm{~m} / \mathrm{s}^{2}$ for the east-west component.

\section{Concluding Remarks}

Low-frequency and trend compensation of broadband seismograms is implemented using a digital filter based on the inverse characteristics of the broadband seismometer and by removing an estimated Heaviside-type tilt signal. This method was applied to the east-west component of broadband seismograms recorded using VSE355G3 broadband seismometers at the KSN site of F-net, during the 2011 Tohoku Earthquake $\left(M_{\mathrm{w}} 9.0\right)$. A sharpened velocity waveform could be obtained by broadening the frequency band of the original seismogram and the static displacement could be determined by removing the tilt signal from the frequency-compensated seismogram. In addition to tilt motion generated by earthquake faulting, trend signals in broadband seismograms are sometimes the result of local site effects (Kinoshita, 2008; Kinoshita et al., 2009) or nonideal responses of the seismometers. Broadband seismometers may record effects that resemble a tilt of the pier supporting the mass-spring system, but are actually due to internal temperature changes in the instrument. Nonlinearities in the electrical response of the instrumentation and in the mechanical response of the boom suspension resilience during strong motion may also give rise to signals at fre- quencies of less than $0.01 \mathrm{~Hz}$. However, such extraneous signals are beyond the scope of the present study.

Acknowledgments. The author is grateful to Franz Egli, Streckeisen $\mathrm{GmbH}$, and an anonymous reviewer for carefully reviewing the manuscript. The author thanks the NIED for providing the F-net broadband data.

\section{References}

Boore, D. M., Effect of baseline corrections on displacements and response spectra for several recordings of the 1999 Chi-Chi, Taiwan, Earthquake, Bull. Seismol. Soc. Am., 91(5), 1199-1211, 2001.

Hutt, C. R., J. R. Evans, and I. Yokoi, A brief test of the Tokyo Sokushin VSE-355G3 strong-motion velocity meter, Open-file Report 2008-1311, 44 pp., USGS, 2008.

Javelaud, E. H., T. Ohmachi, and S. Inoue, A quantitative approach for estimating coseismic displacements in the near field from strong-motion accelerographs, Bull. Seismol. Soc. Am., 101(3), 1182-1198, 2011.

Kalman, R. E., A new approach to linear filtering and prediction, J. Basic Eng. Trans. ASME, 82, 35-45, 1960.

Kinoshita, S., Tilt measurement using broadband velocity seismograms, Bull. Seismol. Soc. Am., 98, 1887-1897, 2008.

Kinoshita, S. and M. Takagishi, Generation and propagation of static displacement estimated using KiK-net recordings, Earth Planets Space, 63, 779-783, 2011.

Kinoshita, S., H. Ishikawa, and T. Satoh, Tilt motions recorded at two WISE sites for the 2003 Tokachi-Oki Earthquake (M 8.3), Bull. Seismol. Soc. Am., 99(2B), 1251-1260, 2009.

Kitagawa, G., Introduction to Time Series Modeling, 296 pp., Chapman and Hall/CRC, New York, 2010.

Kitagawa, G. and W. Gersch, Smoothness Priors Analysis of Time Series, 261 pp., Springer-Verlag, New York, 1996.

Lee, R. C. K., Optimal Estimation, Identification, and Control, 152 pp., Research Monograph 28, MIT Press, Cambridge, 1964.

Pillet, R. and J. Virieux, The effects of seismic rotations on inertial sensors, Geophys. J. Int., 171(3), 1314-1323, 2007.

Rauch, H. E., F. Tung, and C. T. Striebel, Maximum likelihood estimates of linear dynamic systems, AIAA J., 3, 1444-1450, 1965.

$\mathrm{Wu}$, Y.-M. and C.-F., Wu, Approximate recovery of coseismic deformation from Taiwan strong-motion records, J. Seismol., 11, 159-170, 2007.

S. Kinoshita (e-mail: kkk001@yokohama-cu.ac.jp) 